\title{
Successful reintroduction of methotrexate after pneumonitis in two patients with rheumatoid arthritis
}

\author{
N J Cook, G J Carroll
}

\begin{abstract}
Two patients are described with severe and progressive rheumatoid arthritis in whom methotrexate was reintroduced despite previous methotrexate related pneumonitis. In both patients a marked improvement in disease control occurred without a recurrence of the pneumonitis.
\end{abstract}

In the treatment of severe erosive arthritis, a limited number of disease modifying drugs are available. When all avenues of treatment have led to intolerance or inefficacy in the patient with progressive disease, the question arises as to whether a previously useful but poorly tolerated drug can be safely reintroduced. Factors influencing this decision include the nature of the toxicity previously encountered, the severity of the patient's disease, and the current state of knowledge with respect to the agent in question.

We report here two patients with methotrexate pneumonitis in whom the drug was subsequently reintroduced and in whom pneumonitis did not recur.

\section{Patients and methods \\ PATIENTS}

For the purpose of diagnosis in this study, we used the criteria for methotrexate pneumonitis described by Searles and McKendry.

\section{METHODS}

Gallium-67 scintigraphy was used to monitor the patients during rechallenge with methotrexate. This is a very sensitive but non-specific test for pulmonary inflammation which has been shown to correlate well with other indices of disease activity in inflammatory disorders of the lung, such as sarcoidosis and pulmonary fibrosis. ${ }^{2-4} \mathrm{~A}^{67} \mathrm{Ga}$ scan may be abnormal before symptoms develop or before clinical or radiological signs are apparent. ${ }^{56}$

The ${ }^{67} \mathrm{Ga}$ index used was based on the method described by Line $e t a l^{2}$; the maximum possible gallium index is $400 \mathrm{U}$ and a scan is considered to be abnormal if the index is greater than $50 \mathrm{U}$.

\section{Results}

PATIENT 1

A 69 year old woman with a 40 year history of rheumatoid arthritis characterised by widespread joint erosion and high concentrations of rheumatoid factor and antinuclear antibodies was treated with methotrexate, $2.5 \mathrm{mg}$ intra- muscularly once a week, beginning in May 1983 , and increasing to $7.5 \mathrm{mg}$ a week over three months. Previous disease modifying drugs included gold salts, cyclophosphamide, sulphasalazine, D-penicillamine, azathioprine, and levamisole, all of which had been discontinued because of side effects or lack of efficacy. This patient was unable to tolerate prednisolone. Methotrexate was tolerated and its use was accompanied by marked symptomatic improvement.

In April 1985, she was admitted to her local hospital with a three week history of fever, malaise, dyspnoea, and dry cough. She had never smoked but had a past history of mild asthma controlled by regular use of salbutamol. Examination showed respiratory rate $35 /$ minute, pulse rate 120 /minute, blood pressure $140 / 80$ $\mathrm{mmHg}$, temperature $37 \cdot 8^{\circ} \mathrm{C}$ (subsequently peaking at $38 \cdot 1^{\circ} \mathrm{C}$ ). Auscultation showed widespread pulmonary crepitations. A chest radiograph (fig 1) showed diffuse pulmonary infiltrates. Laboratory studies showed a haemoglobin concentration of $111 \mathrm{~g} / \mathrm{l}$, white cell count of $4.5 \times 10^{9} / 1$ (neutrophils $76 \%$, lymphocytes $16 \%$, monocytes $8 \%$ ), and an erythrocyte sedimentation rate of $22 \mathrm{~mm} /$ hour. No bacteria were cultured from three sets of blood cultures.

The patient was treated with intravenous ampicillin, gentamicin, and nebulised salbutamol. Her condition deteriorated and she was transferred to an intensive care unit where her arterial blood gas tensions were: $\mathrm{PCO}_{2} 30$ $\mathrm{mmHg}, \mathrm{Po}_{2} 48 \mathrm{mmHg}$ on $14 \mathrm{l}$ oxygen/min $(\mathrm{pH}$

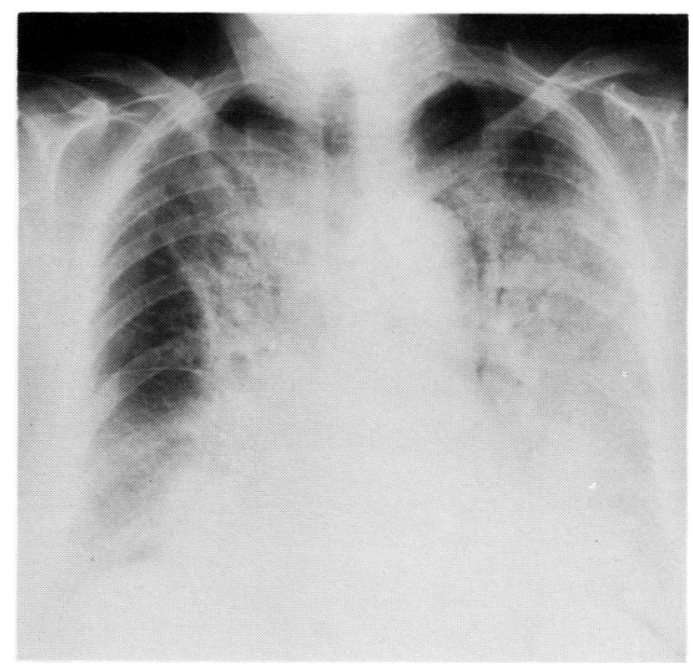

Figure 1 Chest radiograph of patient 1 on presentation in an erect position. 
$7 \cdot 41$, hydrogen carbonate $19 \mathrm{mmol} / \mathrm{l}$, saturation $85 \%)$.

A Swan Ganz catheter was inserted and showed low pulmonary artery wedge pressures, excluding pulmonary oedema (pulmonary artery wedge pressure $5 \mathrm{mmHg}$ and pulmonary artery end diastolic pressure $5 \mathrm{mmHg}$ ). Blood and sputum cultures were consistently negative, as were serological tests for viral infection, fungal agents, and legionella.

Methotrexate and all other drugs (paracetamol, prochlorperazine, carbamazepine, and salbutamol) were discontinued and intravenous erythromycin, co-trimoxazole, and cefotaxime were given. Ventilation and inotropic support were subsequently required. Antibiotics were discontinued after 11 days.

The patient improved steadily and two weeks after admission her chest radiograph was normal (fig 2). Over the next two years she had no further symptoms of respiratory disease and her chest radiograph remained normal. There was, however, a steady increase in the activity of the synovitis which confined her to bed. In July 1987, at the patient's request, 2.5 mg of methotrexate was given by mouth once a week. A baseline ${ }^{67} \mathrm{Ga}$ scan at that time was normal. Since then ${ }^{67} \mathrm{Ga}$ scans every six months have shown no evidence of recurrent pneumonitis. She is now maintained on a dose of $3.75 \mathrm{mg}$ methotrexate by mouth, alternating with $5 \mathrm{mg}$ weekly. Her synovitis remains under reasonable control and she leads an independent life, mostly in an electric wheelchair.

\section{PATIENT 2}

In November 1984 a 66 year old woman with a 14 year history of rheumatoid arthritis characterised by widespread erosive joint destruction and high concentrations of rheumatoid factor and antinuclear antibodies was treated with methotrexate $7.5 \mathrm{mg}$ weekly by mouth. Previous disease modifying drugs included intramuscular gold, D-penicillamine, chlorambucil, and azathioprine, all of which had been discontinued because of inefficacy or adverse side effects. Methotrexate induced clinical remission.

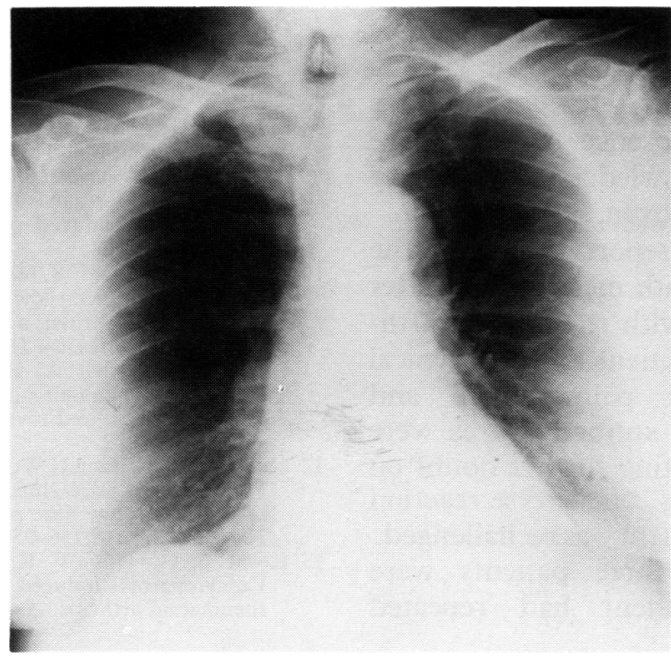

Figure 2 Chest radiograph of patient 1 after recovery in a sitting position. Anteroposterior view.
In August 1986 she was admitted with a three week history of cough, increasing shortness of breath, yellow sputum and mouth ulcers. She had never smoked and had no previous history of respiratory disease. In addition to methotrexate she was receiving ibuprofen, folic acid, diazepam, fluoride, calcium supplements, and an antacid. Examination showed a fever of $38.5^{\circ} \mathrm{C}$, pulse 120 /minute, blood pressure $140 / 70 \mathrm{mmHg}$. Examination of the respiratory system showed a respiratory rate of 24 /minute and decreased expansion bilaterally, but no added sounds. A chest radiograph showed a longstanding blunting of the costophrenic angles but no interstitial abnormality.

Haematological results showed haemoglobin $85 \mathrm{~g} / \mathrm{l}$, white blood cell count $4 \cdot 1 \times 10^{9} / 1$, (neutrophils 65\%, lymphocytes $8 \%$, monocytes $5 \%$, eosinophils $8 \%$ ), platelets $123 \times 10^{9} / 1$. The erythrocyte sedimentation rate was $87 \mathrm{~mm} /$ hour. Urea, electrolytes, and liver function tests were normal. No pathogenic bacteria were grown from throat swabs, sputum cultures, and numerous blood cultures, although the patient had received courses of amoxycillin and minocycline prior to admission. All drugs were stopped and in view of the pancytopenia and mouth ulcers folinic acid $25 \mathrm{mg}$ four times a day was given; no further antibiotics were used.

Over the next 12 days there was a swinging fever to a maximum of $39 \cdot 0^{\circ} \mathrm{C}$ associated with several episodes of diaphoresis. The mouth ulcers persisted for 10 days, but the cough gradually abated and the dyspnoea improved. The mild pancytopenia resolved. A ${ }^{67} \mathrm{Ga}$ scan 14 days after admission showed an intense and diffuse increase in uptake, highly suggestive of interstitial lung disease (gallium index, 242; upper limit of normal subjects, 50). Four weeks later she was discharged with minimal residual dyspnoea.

Repeat ${ }^{67} \mathrm{Ga}$ scans six months and three years later showed an improvement (gallium index 124 and 114, respectively). Respiratory function tests performed at these times were stable and consistent with mild background interstitial lung disease (carbon monoxide transfer factor ( $\mathrm{T}_{\mathrm{L}} \mathrm{CO}$ ) $14.2 \mathrm{ml} / \mathrm{min} / \mathrm{mmHg}$, normal range $18 \cdot 7-25 \cdot 9 \mathrm{ml} / \mathrm{min} / \mathrm{mmHg}$ ).

By March 1987 her arthritis had deteriorated considerably. Methotrexate was reintroduced at a dose of $2.5 \mathrm{mg}$ weekly by mouth and increased over one month to $5 \mathrm{mg}$ weekly. Clinical improvement was evident within three months and has been maintained for three years. There has been no recurrence of the symptoms of pneumonitis and regular respiratory function tests and ${ }^{67} \mathrm{Ga}$ scans have remained stable.

\section{Discussion}

Pneumonitis is an uncommon but potentially life threatening complication of treatment with methotrexate. ${ }^{78}$ It is not known how often pulmonary toxicity occurs when methotrexate is given to patients with rheumatoid arthritis, but in a retrospective study of 92 patients receiving methotrexate prescribed for malignancy, psoriasis, or connective tissue disease, the incidence was found to be $7 \cdot 6 \% .^{9}$ No correlation 
has been shown between pneumonitis and the cumulative dose of methotrexate. Interestingly, pneumonitis has been reported after a total dose of only $12.5 \mathrm{mg}$ methotrexate. ${ }^{10}$

It is not unusual for methotrexate pneumonitis to resolve without the use of corticosteroid treatment, and although steroids are frequently prescribed there is no convincing evidence that they affect the outcome. ${ }^{9}$

Clinical recovery from methotrexate pneumonitis is usually complete, as is the resolution of the changes in chest radiographs, ${ }^{11}$ but there may be persistent abnormalities in pulmonary function. ${ }^{9}$ The death rate from methotrexate pneumonitis is about $10 \%$ when the drug is used in non-rheumatological diseases. ${ }^{9}$

Searles and McKendry ${ }^{i}$ described nine criteria for the diagnosis of methotrexate pneumonitis: (a) acute onset of shortness of breath; (b) fever $>38.0^{\circ} \mathrm{C}$; (c) tachypnoea $\geqslant 28 /$ min and a non-productive cough; $(d)$ radiological evidence of pulmonary interstitial or alveolar infiltrates; $(e)$ white cell count $\leqslant 15.0 \times$ $10^{12} / 1 ;(f)$ negative blood and sputum cultures (obligatory); $(g)$ pulmonary function tests showing restrictive pulmonary function with decreased diffusion capacities; $(h) \mathrm{Po}_{2}<55$ $\mathrm{mmHg}$ on room air at time of admission; and (i) histopathology consistent with bronchitis or interstitial pneumonitis. They considered that a patient who fulfilled six of the nine criteria had definite pneumonitis, five of nine had probable pneumonitis, and four of nine possible pneumonitis.

Patient 1 satisfied seven of the nine criteria (acute dyspnoea, fever, dry cough and tachypnoea pulmonary infiltrates, blood leucocyte concentration $<15.0 \times 10^{12} / 1$, negative cultures, and arterial hypoxaemia $\left(\mathrm{Po}_{2}<60 \mathrm{mmHg}\right)$. Accordingly this patient may be classified as definite. Patient 2 satisfied four of the nine criteria (sudden dyspnoea, fever, blood leucocyte concentration $<15 \cdot 0 \times 10^{12} / 1$, and negative cultures). However, the striking scintigraphic evidence of lung inflammation provides additional support for drug related pneumonitis. Furthermore, advancing interstitial lung disease has not been observed during the subsequent three years. We consider that patient 2 should be classified as probable methotrexate pneumonitis, but concede that pulmonary infection may have been responsible. The diagnostic criteria advocated by Searles and McKendry ${ }^{1}$ have limitations and in the absence of supportive histopathology it is acknowledged that neither case can be considered proven.

There are two previous reports describing the outcome of rechallenge with methotrexate after pneumonitis in patients with rheumatoid arthritis. In one report the patient had an atypical presentation with chest pain, cough and dyspnoea. ${ }^{1}$ Parainfluenza antibody titres were raised in this patient, casting further doubt on the diagnosis. Nonetheless, no adverse reaction was observed when this patient was rechallenged.

In the other report three patients were rechallenged. $^{12}$ One patient had repeated episodes of respiratory illness each time methotrexate was reinstated and the diagnosis was eventually made after an open lung biopsy. The other two patients did not develop recurrent pneumonitis after the reintroduction of methotrexate.

Successful reintroduction of methotrexate after drug related pancytopenia in rheumatoid arthritis has also been described. ${ }^{13}$

A large survey is required to identify those patients at particular risk of developing pneumonitis while receiving methotrexate. Until then it is neither practicable nor of established value to perform regular respiratory function tests on all patients receiving methotrexate. Patients who resume methotrexate after pneumonitis are clearly a high risk group and in this situation ${ }^{67} \mathrm{Ga}$ scintigraphy may be a useful adjunct to respiratory function tests for noninvasive surveillance.

The two patients described here and those reported elsewhere indicate that methotrexate can sometimes be safely reintroduced following pulmonary toxicity in patients with rheumatoid arthritis. However, reports of recurrent pneumonitis on rechallenge emphasise that this is not always possible and caution is therefore recommended.

We thank Dr E T Owen for permission to report on his patien and for his helpful comments. We also thank Ms A Dalgarno for her secretarial services.

1 Searles G, McKendry R J R. Methotrexate pneumonitis in rheumatoid arthritis: potential risk factors. Four case reports and $1164-70$.

2 Line B R, Fulmer J D, Reynolds H Y, et al. Gallium-67 citrate scanning in the staging of idiopathic pulmonary fibrosis: correlation with physiological and morphologic features and bronchoalveolar lavage. Am Rev Respir Di 1978; 118: 355-65

3 Line B R, Hunninghake G W, Keogh B A, Jones A E, Johnston G S, Crystal R G. Gallium-67 scanning to stage the alveolitis of sarcoidosis; correlation with clinical studies, pulmonary function studies and bronchoalveolar lavage. Am Rev Respir Dis 1981; 123: 440-6.

4 Crystal R G, Gadek J E, Ferrans V J, Fulmer J D, Line B R, Hunninghake $G \mathbf{W}$. Interstitial lung disease; current concepts of pathogenesis, staging and therapy. Am $\mathcal{F}$ Med 1981;70: 542-68.

5 MacMahon $\mathrm{H}$, Bekerman $\mathrm{C}$. The diagnostic significance of gallium 67 lung uptake in patients with normal chest gallium 67 lung uptake in patients with
radiographs. Radiology 1978; 127: 189-93.

6 Richman S D, Levenson S M, Bunn P A, Flinn G S Johnston G S, DeVita V T. Gallium 67 accumulation in pulmonary lesions associated with bleomycin toxicity. Cancer 1975; 36: 1966-72.

7 Goldman G C, Moschella S L. Severe pneumonitis occurring during methotrexate therapy. Arch Dermatol 1971; 103: 194-7.

8 Gispen J G, Alarcon G S, Johnson J J, Acton R T, Barger B O, Koopman W J. Toxicity to methotrexate in rheumatoid arthritis. F Rheumatol 1987; 14: 74-8.

9 Sostman H D, Matthay R A, Putman C E, Walker Smith G J Methotrexate induced pneumonitis. Medicine (Baltimore) 1976; 55: 371-88.

10 Ridley M G, Wolfe C S, Mathews J A. Life threatening acute pneumonitis during low dose methotrexate treatment for pneumonitis during low dose methotrexate treatment for rheumatoid arthritis: a case report and
literature. Ann Rheum Dis 1988; 47: 784-8.

11 Green L, Schattner A, Berkenstadt H. Severe reversible interstitial pneumonitis induced by low dose methotrexate; interstitial pneumonitis induced by low dose methotrexate; report of a case

12 Carson C W, Cannon G W, Egger M J, Ward J R, Clegg D O. Pulmonary disease during the treatment of rheumatoid arthritis with low dose pulse methotrexate. Semin Arthritis Rheum 1987; 16: 186-95.

13 Kevat S G, Hill W R, McCarthy P J, Ahern M J. Pancytopaenia induced by low-dose methotrexate fo rheumatoid arthritis. Am NZ F Med 1988; 18: 697-700. 